\title{
Psicooncología
}

ISSN: $1696-7240$

\section{Validez y confiablidad de la Escala de Apoyo Social Percibido (MSPSS)}

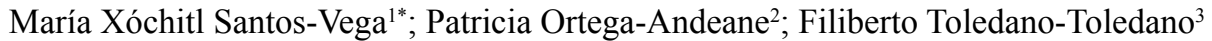

Recibido: 22 de febrero de 2021 / Aceptado: 2 de mayo de 2021

Resumen. Introducción: Existe evidencia de que el apoyo social es una variable fundamental en la investigación y en la intervención con familias en contextos de salud y enfermedad crónica. Objetivo: Obtener las propiedades psicométricas de la Escala de Apoyo Social Percibido (MSPSS) en cuidadores primarios informales de pacientes con cáncer. Método: Estudio instrumental, en el que se entrevistó a 257 cuidadores primarios informales (CPI) que acudían a consulta externa en un Instituto Nacional de Salud en la Ciudad de México para el tratamiento contra el cáncer. Los participantes fueron seleccionados de manera no probabilística, de los cuales 227 eran mujeres $(83,7 \%)$; con un rango de edad de 18 a 65 años $(M=31, D T=9,06)$; el $45,6 \%$ estaban casados, $61,11 \%$ tenían hijos y dedicaban más de 30 horas a la semana al cuidado del paciente. Los cuidadores respondieron un cuestionario de datos sociodemográficos y clínicos, la MSPSS (versión traducida al español) y la Escala de Malestar Psicológico de Kessler (K-10). Se realizó un análisis factorial confirmatorio (AFC) y un análisis de confiabilidad (Coeficiente Alfa de Cronbach). Resultados: El AFC obtuvo índices de ajuste para la MSPSS (CMIN 55,23; GFI 0,997; AGFI 0,995; NFI 0,996; RMR 0,053), y un coeficiente confiabilidad para la escala completa $\alpha=0,908$. Además, se probó la validez de criterio divergente mediante la correlación de los puntajes de la MSPSS y los puntajes de la K-10. Conclusiones: Este estudio aporta evidencias de validez y confiabilidad satisfactorias para la MSPSS y, por tanto, es un instrumento sensible para la medición de apoyo social en poblaciones de cuidadores familiares de pacientes con enfermedades crónicas.

Palabras clave: Apoyo social, validez, confiabilidad, cuidadores primarios, cáncer.

\section{[en] Validity and reliability of Perceived Social Support Scale (MSPSS)}

\footnotetext{
Abstract. Introduction: There is evidence that social support is a fundamental variable in research and intervention with families in the context of health and chronic disease. Aims: Obtain evidence of validity and reliability of the Perceived Social Support Scale (MSPSS) in informal primary caregivers of patients with cancer. Methods: Instrumental study, in which a non-probabilistic sample of 257 informal primary caregivers (CPI) who attended outpatient consultation at a National Institute of Health in Mexico City for cancer treatment was interviewed. Participants were mostly woman $(88,3 \%)$ with ages ranging from 18 to 65 years $(M=31, S D=9,06)$; were married $(45,6 \%)$ and approximately

1 María Xóchitl Santos-Vega. Facultad de Psicología Universidad Nacional Autónoma de México. México.

Email: vega919@hotmail.com

2 Patricia Ortega- Andeane. División de Posgrado y de Investigación, Facultad de Psicología de la Universidad Nacional Autónoma de México.

Email: patricia.andeane@gmail.com

3 Filiberto Toledano-Toledano. Unidad de Investigación en Medicina Basada en Evidencias, Hospital Infantil de México Federico Gómez Instituto Nacional de Salud.

Email: yosoyfiliunam@gmail.com

* Dircción de correspondencia: María Xóchitl Santos-Vega .Avenida Aztecas Manzana. 91, Lote 9, Colonia Ajusco. Alcaldía de Coyoacán. C. P. 04300. Ciudad de México.

E-mail: vega919@hotmail.com
} 
$61.11 \%$ had children and spent more than 30 hours a week caring for the patient $(61,11 \%)$. Caregivers answered a questionnaire of sociodemographic and clinical data, the MSPSS (translated into Spanish) and the Kessler Psychological Discomfort Scale (K-10). Results: Confirmatory factor analysis showed the following fit indices for the MSPSS: CMIN: 55.23; GFI: 0, 997; AGFI: 0,995; NFI: 0,996; RMR: 0,053 . Reliability coefficient for the full scale was 0,908 . In addition, the validity of divergent criteria was tested by correlating the MSPSS scores and the K-10 scores. Conclusions: This study provides satisfactory validity and reliability evidence for the MSPSS and, therefore, is a useful instrument for measuring social support in populations of family caregivers of patients with chronic diseases.

Keywords: Social support, validity, reliability, primary caregivers, cancer.

Sumario: 1. Introducción 2. Método 2.1 Fase 12.2 Fase 23 Discusión y conclusiones 4. Agradecimientos 5. Referencias bibliográficas

Como citar: Santos-Vega MX, Ortega-Andeane P, Toledano-Toledano F. Validez y confiablidad de la Escala de Apoyo Social Percibido (MSPSS). Psicooncología 2021; 18: 333-345, https://dx.doi. org/10.5209/psic. 77756

\section{Introducción}

El cáncer es una de las enfermedades crónicas no transmisibles con mayores efectos en la vida del paciente y su familia; se ha observado que el impacto cubre las esferas física, emocional, familiar, económica y laboral ${ }^{(1-3)}$. Cuidar de un paciente con enfermedad crónica implica una disminución significativa en las relaciones interpersonales, disminución de apoyo social y la acumulación de estresores ambientales ${ }^{(4)}$.

Frecuentemente, el rol de cuidado lo adopta un miembro de la familia, quien además brinda protección y apoyo para el paciente, esta persona se convierte en el cuidador primario informal (en adelante CPI), se le denomina de esta manera porque no reciben remuneración económica por la ayuda que proporcionan ${ }^{(5-6)}$. En población mexicana se ha reportado que el perfil del CPI informal se asocia con el sexo femenino, mayores de 40 años y con nivel de escolaridad básica ${ }^{(7)}$.

Durante la trayectoria de la enfermedad, los cuidadores primarios requieren de apoyo para satisfacer las necesidades atención médica del paciente y de información relacionada con la enfermedad, además de apoyo emocional y psicológico para ellos mismos ${ }^{(8)}$. En este contexto, el apoyo social representa un recurso que ayuda a prevenir o amortiguar el proceso de estrés. El apoyo social se refiere a las relaciones significativas para un individuo y la evaluación subjetiva que ese individuo hace del apoyo que recibe ${ }^{(9)}$. En tanto, el apoyo social percibido se deriva de una valoración cognoscitiva de estar conectados con otros en quienes se confía y de recibir su ayuda en caso de necesidad ${ }^{(10)}$. De acuerdo con diversos estudios el apoyo social ayuda a disminuir la carga en cuidadores ${ }^{(11-12)}$; en la presencia de sintomatología depresiva ${ }^{(13)}$; y ajuste del cuidador ante la enfermedad de su paciente ${ }^{(14)}$.

Otros hallazgos sugieren que existe una relación negativa entre el estado de salud del paciente con cáncer y la salud de su cuidador ${ }^{(15-16)}$, y que el apoyo social parece jugar un rol muy importante en la calidad de vida de esta población ${ }^{(17)}$. Y son la familia, los amigos y las personas significativas las principales fuentes de apoyo en el proceso de recuperación de la salud, el sentido de identidad, el fomento de conductas saludables y control sobre el ambiente. 
Desde la década de los 70s existía interés por investigar el rol del apoyo social como un recurso potencial de enfrentamiento ante los efectos negativos o estresantes. Así surgió la Escala Multidimensional de Apoyo Social Percibido (MSPSS), que representó una alternativa ante los problemas que implicaba la definición del constructo y las dimensiones que éste debería cubrir. Esta escala se ha caracterizado por ser breve y poseer propiedades psicométricas que permiten evaluar el apoyo emocional recibido. Inicialmente la escala se componía por 12 reactivos, que medían el apoyo en tres importantes dimensiones de la vida individual: familia, amigos y otros significativos ${ }^{(18)}$.

La MSPSS ${ }^{(18)}$ es un instrumento utilizado con frecuencia y cuenta con varias traducciones y validaciones en distintos entornos y poblaciones que no hablan el idioma inglés. Entre ellas destacan las validaciones realizadas con adolescentes ${ }^{(19)}$, pacientes con enfermedades cardiacas ${ }^{(20)}$, pacientes con trastornos mentales graves ${ }^{(21)}$ y cuidadores primarios ${ }^{(22)}$.

En la mayoría de los estudios en los que se han probado las propiedades psicométricas de la MSPSS se han obtenido resultados satisfactorios. Por ejemplo, Dambi y colaboradores ${ }^{(22)}$ realizaron una revisión sistemática en la que evaluaron 22 traducciones de 70 estudios en diferentes países y sus principales resultados indicaron que el método más utilizado en las validaciones fue por conveniencia, con un diseño de estudio transversal $(n=16)$, la mayoría de los estudios se llevaron a cabo en poblaciones de altos ingresos económicos $(n=16)$, y en ambientes clínicos $(n=11)$. Los participantes tenían diferentes edades, sin embargo, se pudo identificar que la edad promedio en jóvenes fue 14,8 años $(D T=1,6)$, y en adultos 58,7 años $(D T=13,2)$.

En entornos de salud, se ha identificado que las fuentes de apoyo social de los cuidadores primarios informales, provienen principalmente de la familia, amigos y otras personas significativas que, incluso pueden ser otros cuidadores o parte del personal médico de los centros de salud ${ }^{(23)}$. Es evidente que el recurso primario de apoyo sea la familia, ya que son las personas en la red social más cercana y con las que crean vínculos afectivos e íntimos. Con base en la revisión de la literatura, se puede observar que la mayoría de las traducciones no utilizaron un método riguroso. Por ejemplo, muchos de los estudios solo tradujeron la versión al idioma de interés y no re tradujeron al idioma original. También, se identificó que tenían poca evidencia de validez estructural, es decir, la mayoría no realizaron Análisis Factorial Confirmatorio (AFC), en muchos casos apenas cumplió el criterio de un alfa de 0,70 y además se requiere evidencia empírica que respalde la robustez psicométrica de las versiones traducidas de la MSPSS, por lo que deben considerarse estos aspectos antes de utilizar una versión traducida. Por ello, el objetivo de este estudio es aportar evidencias de validez y confiabilidad de la Escala MSPSS para su aplicación en la población de cuidadores primarios informales de pacientes con cáncer. Se consideró validar una versión traducida y adaptada de la versión original (inglés) al español, basada en un método adecuado, riguroso y culturalmente relevante.

\section{Método}

Estudio instrumental, transversal, no experimental dividido en dos fases ${ }^{(24)}$. La investigación se realizó previa aceptación del protocolo de investigación por el Comité de Ética e Investigación de un hospital público de alta especialidad. 


\subsection{Fase 1}

El objetivo de esta fase fue traducir y adaptar la MSPSS al español (México), someter esta versión a un jueceo por expertos y aplicarla a una muestra piloto de 30 cuidadores primarios informales de pacientes con cáncer.

\section{Participantes}

Una muestra de 30 cuidadores primarios informales; hombres y mujeres, mayores de 18 años, seleccionados de manera no probabilística intencional ${ }^{(25)}$.

\section{Instrumentos}

a) Cuestionario de datos sociodemográficos y clínicos: Serie de preguntas estructuradas como sexo del cuidador, edad del cuidador, sexo del paciente, edad del paciente (meses), ocupación del cuidador, si tiene o no pareja, padecimientos crónicos del cuidador, tiempo transcurrido desde el diagnóstico del paciente.

b) Escala Multidimensional de Apoyo Social Percibido ${ }^{(18)}$ : Esta versión fue traducida del inglés al español, y retraducida por un tercero experto en el idioma inglés a fin de verificar la precisión de la traducción. La escala se compone de 12 reactivos que miden tres dimensiones del apoyo social percibido: 1) apoyo de otros significativos (ej., Hay una persona especial con la que puedo compartir mis tristezas), 2) apoyo familiar (ej., Mi familia me apoya en lo que necesito), y 3) apoyo amigos (ej., Mis amigos tratan de ayudarme). Los reactivos tienen una escala de respuesta tipo Likert de cinco puntos que van de Nunca (0) a Siempre (5). Para este instrumento se reporta un alfa de Cronbach de 0,72, 0,85, y 0,75, respectivamente, y para la escala completa el alfa es de 0,85 . Los reactivos tienen una escala de respuesta tipo Likert de cinco puntos que van de Nunca (0) a Siempre (5).

\section{Procedimiento}

Se siguió el procedimiento para la construcción y adaptación de instrumentos de medición psicológicos propuesto por Carretero-Dios y Pérez ${ }^{(26)}$ y la obtención de evidencias de validez para pruebas psicométricas ${ }^{(27-29)}$. El primer paso fue traducir la versión original al español (México), se contó con la participación de expertos en los idiomas inglés y español. Posteriormente, se hizo una re-traducción del español al idioma original de la escala (inglés), nuevamente se recurrió a expertos traductores, cuya lengua materna es el inglés. Después, se analizaron las posibles discrepancias entre las traducciones de los reactivos en un comité de expertos que dominen ambos idiomas. Como resultado se obtuvo una primera versión del MSPSS conformada por 12 reactivos y siete opciones de respuesta. Esta versión fue evaluada en los rubros: formato de la escala original, instrucciones, aplicación y opciones de respuesta para la población meta. Los expertos consideraron que algunos reactivos de la escala evaluaban dos aspectos en un mismo enunciado, por lo que sugirieron separar las ideas en oraciones distintas. 


\section{Resultados de la evaluación por jueces expertos}

Basados en los resultados obtenidos de las evaluaciones por jueces expertos, se determinó someter a un piloteo la versión de la Escala Multidimensional de Apoyo Social Percibido con 15 reactivos y no 12 como en la versión original. Esta decisión se fundamentó a partir de las primeras observaciones que se realizaron a la escala, en las que los jueces encontraron que los reactivos dos y nueve, medían dos ideas en el mismo reactivo, por lo que era justificable psicométricamente separar las ideas en oraciones diferentes. Posteriormente, la versión de la escala fue evaluada por siete jueces expertos en psicología en las áreas de la salud, clínica y ambiental. Entre las observaciones de los jueces se encontraban que el número de opciones de respuesta de la versión original eran demasiadas, difíciles de comprender y poco atractivas para la población objetivo, una razón de la complejidad es la comprensión de estas opciones debido al bajo nivel educativo de dicha población. Así al evitar opciones como "ni de acuerdo ni en desacuerdo" se busca reducir la ambigüedad y en su lugar se recomendó utilizar un formato de respuesta de frecuencia con cinco opciones (de nunca a siempre).

Para probar esta hipótesis, se llevaron a cabo dos estudios piloto en los que participaron 30 cuidadores primarios de pacientes con cáncer de un hospital público de alta especialidad (15 para el primer piloteo y 15 para el segundo), la edad de los cuidadores oscilaba entre 18 y 60 años. En el primer piloteo se les aplicó la versión de la escala que contenía 15 reactivos y las siete opciones de respuesta propuestas originalmente: Totalmente en desacuerdo (0), Muy en desacuerdo (1), En desacuerdo (3), Ni de acuerdo ni en desacuerdo (4), De acuerdo (5), Muy de acuerdo (6) y Totalmente de acuerdo (7). En el segundo piloteo se les aplicó la versión de la escala que contenía 15 reactivos y cinco opciones de respuesta como sugirieron los jueces: Nunca (1), Casi nunca (2), A veces (3), Casi siempre (4) y Siempre (5).

Los resultados del estudio piloto indicaron que los cuidadores tuvieron mayor dificultad para diferenciar los significados o la magnitud entre las opciones de respuesta de la primera versión, incluso la última opción de respuesta no fue clara para los participantes; mientras que en el segundo piloteo, los participantes se mostraron más seguros al elegir entre las opciones de respuesta y no cuestionaban a qué se hacía referencia. En consecuencia, se decidió optar por la segunda versión ya que era la más adecuada para ser aplicada a la población objetivo. Una vez conformada la escala con los reactivos y las opciones de respuesta, se procedió a seguir la metodología propuesta por Downing ${ }^{(29)}$, para la elaboración de una prueba, y la obtención de evidencias de validez y confiabilidad.

La segunda versión de la MSPSS (15 reactivos) fue aplicada de manera individual a 30 cuidadores primarios informales de pacientes con cáncer que se encontraban en la sala de espera del hospital. Los participantes fueron evaluados por el investigador, quien les explicó el objetivo de la investigación; les leyó el consentimiento informado y les comentó que el llenado del cuestionario les llevaría 10 minutos. Si accedían a participar, se les pedía que firmaran el consentimiento informado, garantizándoles completa confidencialidad de sus respuestas, asimismo, se les exponía que su participación en la investigación no comprometía de ninguna manera el tratamiento que recibía su paciente y que tenían completa libertad para abandonar el estudio si así lo disponían. Al finalizar la aplicación se les otorgaba un agradecimiento verbal por su 
participación en el estudio. Derivado de esta fase, se hicieron los ajustes necesarios a la primera versión adaptada de la MSPSS y se obtuvo la versión B.

\subsection{Fase 2}

El objetivo de esta fase fue aportar evidencias de validez y confiabilidad para la versión traducida y adaptada de la MSPSS.

\section{Participantes}

Una muestra total de 257 cuidadores primarios informales, seleccionados de manera no probabilística intencional $^{(25)}$ de los cuales 227 eran mujeres y 30 hombres, con un rango de edad de 18 a 65 años $(M=35,28$ años, DT =9,06). El 45,6\% de los participantes estaban casados, $35,6 \%$ vivían en unión libre, $11,1 \%$ solteros y 7,8\% divorciados. 62 , $2 \%$ reportaron tener educación básica, $17,8 \%$ media superior, $5,6 \%$ superior y $14,4 \%$ mencionaron no tener estudios. El 57, $8 \%$ de los cuidadores primarios eran amas de casa, $30 \%$ empleados y $12,2 \%$ se encontraban desempleados. Los participantes fueron entrevistados en la sala de espera de un hospital público en el año 2019. Los criterios para ser incluidos en el estudio fueron: tener un paciente con diagnóstico de cáncer en cualquier etapa evolutiva, tener más de seis meses desempeñando el rol de cuidador primario, ser mayor de 18 años y que de manera voluntaria aceptaran participar en el estudio mediante la firma del consentimiento informado.

\section{Instrumentos}

a) Cuestionario de datos sociodemográficos y clínicos: Se utilizó el cuestionario de datos sociodemográficos citado en la fase uno.

b) Escala Multidimensional de Apoyo Social Percibido ${ }^{(18):}$ En su versión traducida y adaptada. La escala se compone de 15 reactivos que miden tres dimensiones del apoyo social percibido: 1) apoyo de otros significativos (ej., Hay una persona especial con la que puedo compartir mis tristezas), 2) apoyo familiar (e. g., Mi familia me apoya en lo que necesito), y 3 ) apoyo amigos (ej., Mis amigos tratan de ayudarme). Los reactivos tienen una escala de respuesta tipo Likert de cinco puntos que van de Nunca (0) a Siempre (5). Los reactivos tienen una escala de respuesta tipo Likert de cinco puntos que van de Nunca (0) a Siempre (5).

b) Escala de Malestar Psicológico versión en español ${ }^{(30)}$ : Es un instrumento de autorreporte compuesta por 10 reactivos, unifactorial que mide el malestar psicológico (ej., Con qué frecuencia. ¿Se ha sentido cansado sin motivo?, ¿Se ha sentido nervioso?, ¿Se ha sentido deprimido (a)?) Los reactivos tienen una escala de respuesta tipo Likert de cinco puntos que van de Nunca (0) a Siempre (5).

\section{Procedimiento}

Los participantes fueron entrevistados por el investigador en una sala de espera de un hospital público en el año 2019. A cada uno de los participantes se les explicó el objetivo de la investigación; se les leyó el consentimiento informado y se les comentó que el llenado del cuestionario les llevaría aproximadamente 15 minutos. Si accedían a participar, se les entregaba la carta de consentimiento informado 
para otorgar por escrito su autorización y firma, y la batería de instrumentos que contenía un cuestionario de datos sociodemográficos, el MSPSS y la escala $\mathrm{K}-10$. Al firmar este documento, se garantizó la completa confidencialidad de sus respuestas, asimismo, se les expuso que su participación en la investigación no comprometía de ninguna manera el tratamiento que recibía su paciente y que tenían completa libertad para abandonar el estudio si así lo disponían. Al finalizar la aplicación se les otorgaba un agradecimiento verbal por su participación en el estudio y si lo deseaban, se les informarían los resultados de su evaluación.

\section{Resultados fase 2}

\section{Evidencias de validez factorial}

Los datos se analizaron mediante el paquete estadístico SPSS versión 20. Primeramente, se realizó un análisis de discriminación de reactivos y se pudo observar que, los reactivos 10, 14 y 15 no discriminaron, por tanto, fueron excluidos en los siguientes análisis. Posteriormente, a fin de probar la estructura teórica de la escala, se efectuó un Análisis Factorial Exploratorio (AFE), con el método de factorización de ejes principales y rotación Oblimin. Se empleó el AFE puesto que brinda buenos resultados con escalas de respuesta con cinco o más opciones ${ }^{(30)}$. El AFE efectuado abrió un valor de la medida de adecuación muestral de Kaiser-Meyer-Olkin (KMO) de 0,891, la significancia de la prueba de esfericidad de Bartlett fue $\mathrm{p}<0,001$, ambos satisfactorios. En el AFE se identificaron tres factores cuyo porcentaje de varianza explicada fue 77, $41 \%$. En la tabla 1 se muestra la solución y las cargas factoriales para cada uno de los reactivos, así como la media y la desviación estándar de los puntajes promedio para la escala total, los cuales se ubicaron por arriba de la media teórica.

Tabla 1. Reactivos de la MSPSS con sus respectivas cargas factoriales y descriptivos.

\begin{tabular}{|c|c|c|c|c|c|}
\hline Factor & Reactivo & $\begin{array}{l}\text { Carga } \\
\text { factorial } \\
\text { (AFE) }\end{array}$ & Media & DT & $\begin{array}{l}\text { Carga } \\
\text { factorial } \\
(\mathrm{AFC})\end{array}$ \\
\hline \multirow[t]{5}{*}{$\begin{array}{l}\text { Otros } \\
\text { significativos }\end{array}$} & $\begin{array}{l}\text { 3. Hay una persona especial con la } \\
\text { que puedo compartir mis tristezas. }\end{array}$ & 0,957 & 4,35 & 1,15 & 0,844 \\
\hline & $\begin{array}{l}\text { 2. Hay una persona especial con la } \\
\text { que puedo compartir mis alegrías }\end{array}$ & 0,920 & 4,45 & 1,09 & 0,899 \\
\hline & $\begin{array}{l}\text { 4. Tengo una persona especial que es } \\
\text { una fuente de consuelo para mí. }\end{array}$ & 0,916 & 4,34 & 1,20 & 0,876 \\
\hline & $\begin{array}{l}\text { 1. Hay una persona especial que está } \\
\text { cerca de mi cuando la necesito }\end{array}$ & 0,776 & 4,32 & 1,16 & 0,847 \\
\hline & $\begin{array}{l}\text { 5. Hay una persona especial en mi } \\
\text { vida que se preocupa por lo que sien- } \\
\text { to. }\end{array}$ & 0,733 & 4,32 & 1,14 & 0,874 \\
\hline
\end{tabular}




\begin{tabular}{llcccc}
\hline Factor & \multicolumn{1}{c}{ Reactivo } & $\begin{array}{c}\text { Carga } \\
\text { factorial } \\
\text { (AFE) }\end{array}$ & Media & $\begin{array}{c}\text { DT } \\
\text { Familia }\end{array}$ & $\begin{array}{c}\text { Carga } \\
\text { factorial } \\
\text { (AFC) }\end{array}$ \\
\hline & $\begin{array}{l}\text { 6. Mi familia trata de ayudarme en lo } \\
\text { que puede. }\end{array}$ & 0,910 & 4,09 & 1,26 & 0,883 \\
& $\begin{array}{l}\text { 7. Mi familia me apoya emocional- } \\
\text { mente }\end{array}$ & 0,901 & 4,00 & 1,38 & 0,876 \\
& $\begin{array}{l}\text { 8. Mi familia me apoya en lo que } \\
\text { necesito. }\end{array}$ & 0,899 & 3,88 & 1,34 & 0,883 \\
& $\begin{array}{l}\text { 9. Puedo hablar de mis problemas } \\
\text { con mi familia. }\end{array}$ & 0,607 & 3,18 & 1,58 & 0,746 \\
\hline Amigos & $\begin{array}{l}\text { 12. Puedo contar con mis amigos } \\
\text { cuando las cosas van mal. }\end{array}$ & 0,939 & 2,98 & 1,54 & 0,924 \\
& $\begin{array}{l}\text { 13. Tengo amigos con quienes puedo } \\
\text { compartir mis alegrías. }\end{array}$ & 0,906 & 3,10 & 1,59 & 0,865 \\
& $\begin{array}{l}\text { 11. Mis amigos tratan de ayudarme. } \\
\text { 11 }\end{array}$ & 0,882 & 2,98 & 1,50 & 0,944 \\
\hline
\end{tabular}

Método de factorización: Ejes principales

Método Rotación: Oblimin.

\section{Análisis Factorial Confirmatorio (AFC)}

Para evaluar el ajuste del modelo obtenido en el AFE, se efectuó un AFC mediante el método de mínimos cuadrados y se obtuvieron los siguientes índices: CMIN 55,23, GFI 0,997, AGFI 0,995, NFI 0,996, RMR 0,053. E1 modelo resultante mostró índices de ajuste satisfactorios. La solución final del MSPSS se muestra en la figura 1.

Figura 1. Modelo Confirmatorio de la MSPSS

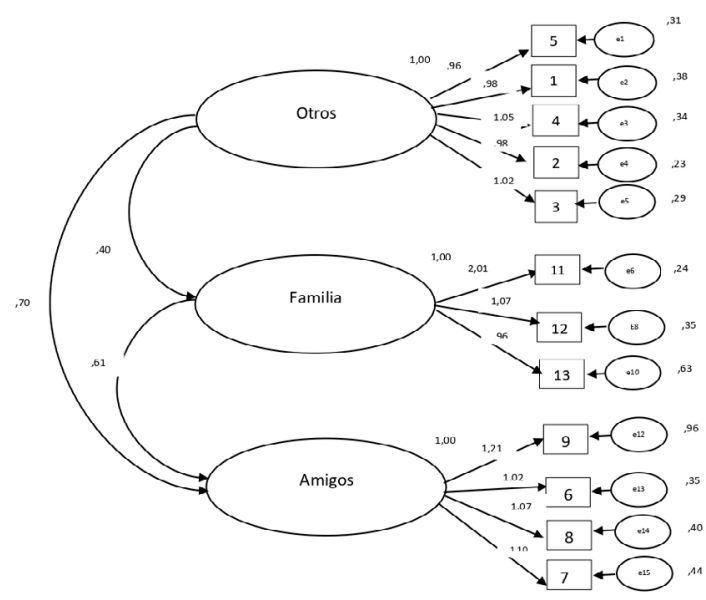


La versión final del Inventario Multidimensional de Apoyo Social Percibido se conformó por 12 reactivos, agrupados en tres factores: otros significativos con cinco reactivos, familia con tres reactivos y amigos con cuatro reactivos.

\section{Consistencia interna}

El índice de consistencia interna obtenida para la escala total fue $\alpha=0,908$ (alfa de Cronbach), considerado un coeficiente de confiabilidad alto. De manera independiente los tres factores: otros significativos $(\alpha=0,942)$, familia $(\alpha=0,907)$ y amigos $(\alpha=$ 0,936) alcanzaron índices altos.

\section{Evidencias de validez de criterio}

Las evidencias de validez de criterio de la Escala Multidimensional de Apoyo Social Percibido se derivaron de su red nomológica, la cual establece asociaciones teóricas entre los constructos apoyo social, y sintomatología ansiosa y depresiva (medidos con la Escala de Malestar Psicológico de Kessler $)^{(18,32)}$. Como se observa en la tabla 2, los análisis de correlación entre los puntajes totales de las escalas MSPPS y K-10, mostraron índices en la dirección esperada $(r=-0,261, p<0,001)$, que resultaron altamente significativos, y evidenciaron la validez discriminante (insertar tabla 2).

Tabla 2. Índices de correlación de la MSPSS y las variables relacionadas en la red nomológica

\begin{tabular}{lccc}
\hline Factor & Media (DT) & Rango & $\mathrm{r}(\leq=0,001)$ \\
\hline MSPSS & $46,64(11,26)$ & 48 & 1 \\
Otras personas significativas & $21,80(5,69)$ & 20 & $0,821^{* *}$ \\
Familiar & $18,0(4,84)$ & 16 & $0,847^{* *}$ \\
Amigos & $9,15(4,36)$ & 12 & $0.666^{* *}$ \\
Malestar Psicológico & $14,55(5,22)$ & 26 & $-0,261^{* *}$ \\
\hline
\end{tabular}

**La correlación es significativa al nivel 0,001 .

Interpretaciones de los puntajes

La interpretación de los puntajes se realiza con base en la puntuación total de la escala, es decir, la suma de todos los reactivos, luego se divide entre el número de reactivos, lo que da como resultado una puntuación total promedio a la que se puede hacer referencia por número de las opciones de respuesta.

\section{Discusión y conclusiones}

Con base en los resultados obtenidos, se pudo apreciar que la Escala Multidimensional de Apoyo Social Percibido es un instrumento con propiedades psicométricas adecuadas y confiables para su aplicación con cuidadores primarios de pacientes con cáncer 
en México. Esta versión de la MSPSS obtuvo propiedades psicométricas similares a otras versiones en español ${ }^{(21)}$. Además, esta versión de la escala aporta evidencias de validez de contenido, criterio constructo y confiabilidad alta. Al igual que en la versión original ${ }^{(18)}$, se obtuvo una estructura de tres factores, comprensible, consistente y parsimoniosa.

La validez de contenido, criterio y constructo de la MSPSS se basó en un instrumento que permite medir otros constructos relacionados teóricamente. De esta manera, el apoyo social se relacionó negativa y significativamente con los síntomas de ansiedad y depresión evaluados con la Escala de Malestar Psicológico, es decir que, a mayor apoyo social, menor percepción de malestar psicológico en las personas, estos datos también son consistentes con hallazgos reportados en la literatura ${ }^{(13)}$. También, se obtuvieron correlaciones altas y significativas entre los tres factores de la MSPSS (otros significativos, familiar y amigos), y los puntajes totales de la escala, lo que significa que los factores son parte del mismo constructo.

Este estudio tiene fortalezas como lo son las evidencias de validez y confiabilidad en una muestra de cuidadores primarios de pacientes con cáncer, que si bien se ha validado en esta población ${ }^{(22)}$, la versión disponible no está traducida al español y no reporta validez convergente o discriminante. En contraparte, esta versión de la escala MSPSS cuenta con evidencias de validez discriminante y apoya la idea de que el apoyo social guarda una relación teórica y empírica con la sintomatología ansiosa y depresiva ${ }^{(33)}$. Contar con un instrumento válido y confiable para esta población representa una oportunidad para detectar las necesidades de apoyo que, en muchas ocasiones son desconocidas para los profesionales de la salud y que dificultan el buen desempeño del rol de cuidado, y traen consigo repercusiones psicológicas para los cuidadores $^{(34)}$.

En contextos de cáncer, la salud del cuidador se ve disminuida y su rol trasciende al apoyo físico, económico, emocional y en algunos casos instrumental al paciente. Asimismo, se ha observado que, bajo estas circunstancias, los cuidadores se convierten en muchos casos en la principal fuente de apoyo para otros cuidadores, con quienes llegan a formar lazos de amistad ${ }^{(23)}$. Por ello es importante fijar la mirada hacia los que de manera "informal" son una pieza clave para la recuperación del paciente. De esta manera, contar con un instrumento que identifique las necesidades de apoyo en esta población, cobra relevancia y brinda a los profesionales de la salud información valiosa para detectar y atender intervenciones oportunas en los cuidadores que más lo necesiten.

Aun cuando esta versión de la Escala Multidimensional de Apoyo Social Percibido obtuvo propiedades psicométricas adecuadas, se considera pertinente tomar en cuenta las limitaciones del estudio, ya que la muestra que se utilizó no fue seleccionada con un método riguroso y no contempló a cuidadores primarios de pacientes con diferentes tipos de cáncer, en edad adulta y en diferentes entidades del país. Por ello, se recomienda que en próximos estudios se amplíe el tamaño de la muestra y contemple las características antes mencionadas para que pueda ser representativa de la población objetivo.

\section{Agradecimientos}

Al Consejo Nacional de Ciencia y Tecnología (CONACYT) por el financiamiento para realizar este estudio, número de becario CVU 548390. 
Este trabajo es parte del proyecto de investigación HIM/2013/019/SSA.1141 Medición y evaluación de la resiliencia ante la enfermedad crónica pediátrica.

\section{Referencias biliográficas}

1. Girgis A, Lambert S, Johnson J, Waller A, Currow D. Physical, psychosocial, relationship and economic burden of caring for people with cancer: a review. J Oncol Pract 2013; 197202. https://doi.org/10.1200/JOP.2012.000690

2. Rokacha A, Findlerc L, Chind J, Leve S, Kollender Y. Cancer patients, their caregivers and coping with loneliness. Psychol Health Med 2013; 18: 135-44. https://doi.org/10.108 $0 / 13548506.2012 .689839$

3. PDQ Supportive and Palliative Care Editorial Board. Family caregivers in cancer roles and challenges. 2017 [Acceso 23 de enero 2020] Disponible en: https://www.ncbi.nlm. nih.gov/books/NBK65845/

4. Raina P, O'Donnell M, Rosenbaum P, Brehaut J, Walter SD., Russell D, et al. The health and well-being of caregivers of children with cerebral palsy. Pediatrics 2005; 115: 62636. https://doi.org/10.1542/peds.2004-1689.

5. Flores ME, Fuentes HL, González GJ, Meza IJ, Cervantes GA, Valle MA. Características principales del cuidador primario informal de adultos mayores hospitalizados. Nure Investigación 2017; 14: 1-16.

6. Vásquez B. La importancia del cuidador en los cuidados paliativos. Informe Médico 2006; 8: 1-4.

7. Galindo O, Meneses A, Herrera A, Cabrera P, Suchil, L, Rivera L, et al. Propiedades psicométricas del Cuestionario General de Salud de Goldberg -GHQ-28- en cuidadores primarios informales de pacientes con cáncer. Psicooncología 2017; 14: 71-81. https:// doi.org/10.5209/PSIC.55812.

8. Sklenarova H, Kruumpelmann A, Haun MW, Friederich HC, Huber J, Thomas M, et al. When Do We Need to Care About the Caregiver? Supportive care needs, anxiety, and depression among informal caregivers of patients with cancer and cancer survivors. Cancer 2015; 121: 1513-9. https://doi.org/10.1002/cncr.29223

9. Escobar MA, Puga D, Martín M. Asociaciones entre la red social y la discapacidad al comienzo de la vejez en las ciudades de Madrid y Barcelona en 2005. Revista Española de Salud Pública 2008; 82: 637-51.

10. Usta Y. Importance of social support in cáncer patients. Asian Pac J Cancer Prev 2012; 13: 3569-72. https://doi.org/10.7314/APJCP.2012.13.8.3569

11. Meecharoen W, Sirapo-ngam Y, Monkong S, Oratai P, Northouse L. Factors influencing quality of life among family caregivers of patients with advanced cancer: A casual model. Pacific Rim Int J Nur 2013; 17:304-16.

12. Ustaalioglu BO, Acar E, Caliska M. The predictive factors for perceived social support among cancer patients and caregiver burden of their family caregivers in Turkish population. Int J Psychiatry Clin Pract 2018; 22: 63-9. https://doi.org/10.1080/13651501.1358370.

13. Sherman DW, McMillan SC. The physical health of patients with advanced pancreatic cancer and the psychological health of their family caregivers when newly enrolled in hospice. J Hospi Paliat Nurs 2015; 17: 235-41. https://doi.org/ 10.1097/ NJH.0000000000000154 
14. Brown J, Gottlieb BH, Maitland SB. Social support and adjustment among wives of men with prostate cancer. J Psychosoc Oncol 2014; 32: 16-36. https://doi.org/10.1080/073473 32.2013.855962.

15. Posluszny DM, Bovbjerg DH, Syrjala K, Agha M, Dew MA. Correlates of anxiety and depression symptoms among patients and their family caregivers prior to allogeneic hematopoietic cell transplant for hematological malignancies. Support Care Cancer 2019; 27: 591-600. https://doi.org/10.1007/s00520-018-4346-3

16. Tan JY, Molassiotis A, Lloyd-Williams M Yorke J. Burden, emotional distress and quality of life among informal caregivers of lung cancer patients: An exploratory study. Eur J Cancer Care 2018; 27. https://doi.org/10.1111/ecc.12691.

17. Warapornmongkhoulkul A, Howteerakul N. Self-efficacy, social support, and quality of life among primary family-member caregivers of patients with cancer in Thailand. J Health Res 2017; 32: 111-22. https://doi.org/ 10.1108/JHR-01-2018-012

18. Zimet GD, Dahlem NW, Zimet SG, Farley GK. La escala multidimensional de apoyo social percibido. J Pers Assess 1988; 52: 30-41. https://doi.org/10.1207/s15327752jpa5201_2

19. Trejos-Herera AM, Bahamón MJ, AlarcónVásquez Y, Vélez JI, Vinaccia S. Validity and reliability of the Multidimensional Scale of Perceived Social Support in Colombian adolescents. Psychosocial Intervention 2018; 27:56-63. https://doi.org 10.5093/pi2018a1

20. Bagherian-Sararoudi R, Hajian A, Ehsan HB, Sarafraz MR, Zimet GD. Psychometric properties of the persian version of the multidimensional scale of perceived social support in Iran. Int J Prev Med 2013; 4:1277-81.

21. Ruiz-Jiménez T, Saiz-Galdós J, Montero-Arredondo MT, Navarro-Bayón D. Adaptación de la Escala Multidimensional de Apoyo Social Percibido en población con trastorno mental grave. Rev Asoc Esp Neuropsiq 2017; 37: 415-37 https://doi.org/10.4321/S021157352017000200006

22. Dambi JM, Tapera L, Chiwaridzo M, Tadyanemhandu C, Nhunzvi C. Psychometric evaluation of the Shona version of the Multidimensional Scale of Perceived Social Support Scale (MSPSS-Shona) in adult informal caregivers of patients with cancer in Harare, Zimbabwe. Malawi Med J 2017; 29: 89-97. https://doi.org/10.4314/mmj.v29i2.3

23. Santos MX. Fuentes de estrés psicoambiental en cuidadores primarios de pacientes crónicos-pediatriatricos [Tesis de Maestría]. México: Universidad Nacional Autónoma de México; 2017.

24. Montero I, Leon OG. A guide for naming research studies in Psychology. Int J Clin Health Psychol 2007; 7: 847-862.

25. Levy PS, Lameshow S. Sampling of populations. Methods and applications. $4^{\text {th }}$ ed. Hoboken, New Jersey: John Wiley \& Sons; 2008.

26. Carretero-Dios H, Pérez C. Standards for the development and review of instrumental studies: Considerations about test selection in psychological research. Int J Clin Health Psychol 2007:7: 863-82.

27. Streiner DL, Norman GR, Cairney J. Health Measurement Scales. A practical guide to their development and use. $5^{\text {th }}$ ed. United Kingdom: Oxford University Press; 2015.

28. DeVellis RF. Scale Development Theory and Applications. $4^{\text {th }}$ ed. University of North Carolina, Chapel Hill; 2017.

29. Downing, SM. Handbook of test development. In D. S. M. Downing \& T. M. Haladyna (Eds). Twelve Steps for Effective Test Development. New Jersey: Lawrence Erlbaum Associates, 2011.

30. Ferrando PJ, Anguiano-Carrasco C. El análisis factorial como técnica de investigación en psicología. Papeles del Psicólogo 2010; 31: 18-33. 
31. Vargas-Terrez B E, Villamil-Salcedo V, Rodríguez- Estrada C, Pérez-Romero J, CortésSotres J. Validación de la escala Kessler 10 (K-10) en la detección de depresión y ansiedad en el primer nivel de atención. Salud Mental 2011; 34: 323-331.

32. Denis A, Callahan S, Bouvard M. Evaluation of the French versión of the multidimensional scale of perceived social support during the postpartum period. Metern Child Health J 2015; 19: 1245-51. https://doi.org/10.1007/s10995-014-1630-9.

33. Götze H, Brähler E, Gansera L, Schnabel A, Gottschalk-Fleischer A, Köhler N. Anxiety, depression and quality of life in family caregivers of palliative cancer patients during home care and after the patient's death. Eur J Cancer Care 2018; 27: e12606. https://doi. org/10.1111/ecc. 12606

34. Ullrich, A, Ascherfeld L, Marx G, Bokemeyer C, Bergelt C, Oechsi K. Quality of life, psychological burden, needs, and satisfaction during specialized inpatient palliative care in family caregivers of advanced cancer patients. BMC Palliat Care 2017; 16: 31. https:// doi.org/10.1186/s12904-017-0206-z. 